also previously shown that protein kinase $C$ (PKC) $\beta$ Il is essential for DC differentiation and that tumor derived factors, particularly IL-6, inhibit DC differentiation by repressing the expression of PKC $\beta$ II through a Stat3 dependent mechanism. However, the pathways activated downstream of PKC $\beta$ II during DC differentiation have yet to be fully characterized. To study this, we used the K562 cell line, which differentiates into DCs with addition of PMA, a known chemical activator of conventional PKC isoenzymes. We have also shown previously that PMA specifically activates PKC 1 II in K562 cells. Addition of PMA to K562 cells showed the activation of the ERK1/2 and NFKB pathways, including both the canonical and non-canonical NFKB pathways. Chemical inhibition of either the ERK $1 / 2$ or NFKB pathway yielded immature DCs with a reduced capacity to stimulate T-cell proliferation when incubated with PMA. Inhibition of ERK1/2 also showed a change in levels of RelB, a NFKB transcription factor, with PMA treatment and during PMA induced DC differentiation. Inhibition of either the ERK $1 / 2$ or NFKB pathways showed an increase in Foxo3a levels, indicating a role for the ERK1/2 and NFKB pathways in Foxo3a regulation during differentiation into mature DCs. Taken together, these results show an integral role for both the ERK and NFKB pathways downstream of PKCBII activation during DC differentiation.

\title{
T Cell Development
}

\section{6}

Spatial interplay between Polycomb and trithorax complexes controls transcriptional activity in T lymphocytes

Onodera, A. ${ }^{1}$, Tumes, D. ${ }^{2}$, Kiuchi, M. ${ }^{1}$, Kokubo, K. ${ }^{1}$, Watanabe, Y. ${ }^{1}$, Hirahara, K. ${ }^{1}$, Kaneda, A. ${ }^{\text {, }}$ Sugiyama, F. ${ }^{3}$, Suzuki, Y. ${ }^{4}$, Nakayama, T. ${ }^{1}$

${ }^{1}$ Chiba University, Chiba, Japan, ${ }^{2}$ South Australian Health and Medical Research Institute, Adelaide, Australia, ${ }^{3}$ University of Tsukuba, Tsukuba, Japan, ${ }^{4}$ University of Tokyo, Kashiwa, Japan

Polycomb group (PcG) and Trithorax group (TrxG) complexes exert opposing effects on the maintenance of transcriptional status, and play a critical role in the expression of developmentally regulated transcription factors through methylation at histone $\mathrm{H} 3-\mathrm{K} 27$ (H3K27me3; a repressive mark) and H3-K4 (H3K4me3; a permissive mark), respectively. However, how they together mediate transcriptional counterregulation remains unknown. Genome-wide analysis revealed that binding of Ezh2 and Menin, central members of the PcG and TrxG complexes, respectively, were reciprocally correlated. Moreover, we identified a developmental change in the fine scale positioning of Ezh2 and Menin in differentiated

T lymphocytes compared to embryonic stem cells (ESCs). Ezh2 and Menin co-occupancy was most frequently observed in ES cells and often lost during development into T lymphocytes. Ezh2-binding upstream and Menin-binding downstream of the transcription start site (TSS) was frequently found at genes with higher transcriptional levels, and Ezh2-binding downstream and Menin-binding upstream was found at genes with lower expression in T lymphocytes. Interestingly, of the Ezh2 and Menin cooccupied genes, those exhibiting occupancy at an identical position displayed greatly enhanced sensitivity to loss of Ezh2. Finally, we also found that different combinations of Ezh2 and Menin occupancy were associated with expression of specific functional gene groups important for $\mathrm{T}$ cell development. Therefore, spatial cooperative gene regulation by the PcG and TrxG complexes may represent a novel mechanism regulating the transcriptional identity of differentiated cells.

\section{7}

Thymic precursors of CD8aa+ intestinal epithelial lymphocytes (IEL) divide into an emigrating and a retained population

Ruscher, R., Kummer, R., Lee, Y.J., Huggenberger, S., Hogquist, K.A.

University of Minnesota, Minneapolis, United States

Intestinal TCRb+CD4-CD8b-CD8a+ (CD8aa) IELs alleviate T cell induced colitis and have been suggested to play a role in virus infection and cancer. Their thymic development has been elucidated 
to some extent, as IEL precursors (IELp) are known to be CD4-CD8-CD5+TCRb+, but is not yet fully understood. Within the thymus, mature IELp were identified based on their expression of CD122 and MHC class I. Two major phenotypic subsets exist within this mature thymic IELp population: a PD1+Tbet- population that preferentially expresses a4b7, and a PD1-Tbet+ population with preferential CD103 expression. These two populations were also distinct in their Valpha repertoire. The PD1+a4b7+ population contains clones that are strongly self-reactive as judged by Nur77GFP and their dramatic increase in Bim deficient mice, while the PD1-Tbet+ population did not show these characteristics. Both gave rise to CD8aa IELs upon adoptive transfer into RAG-/- recipients. However intrathymic labeling revealed that PD1+a4b7+ IELp were the major thymic emigrating population, and emigration was S1P1-dependent. In contrast, PD1-Tbet+ IELp expressed CXCR3, were retained, and accumulated in the thymus with age. Preliminary immunofluorescence data furthermore indicate differential thymic cortico-medullary localization of the IELp subtypes. These experiments more precisely define the behavior of IEL precursors.

\section{4}

Peripheral CD8 T cell receptor revision contributes to the plasticity of the immune repertoire Dash, P. ${ }^{1}$, Wang, G. ${ }^{1}$, Bajracharya, R. ${ }^{1}$, Contento, R. ${ }^{2}$, Mcclaren, J. ${ }^{1}$, Morris, M. ${ }^{1}$, Love, J.C. ${ }^{2}$, Doherty, P. ', Thomas, $P$.

${ }^{1}$ St Jude Children's Research Hospital, Immunology, Memphis, United States, ${ }^{2}$ Koch Institute for Integrative Cancer Research at MIT, Chemical Engineering, Cambridge, United States

Using a technique for the paired amplification of TCR $\alpha$ and $\beta$ chains from single cells, we have discovered a surprising plasticity in the peripheral TCR repertoire, particularly under conditions of inflammation. This plasticity includes multiple instances of TCR revision in the periphery, where changes are made in the TCR coding sequence by de novo rearrangement. Here we describe this process in murine CD8+ T cells derived from influenza-infected lungs and human $T$ cells derived from CMV-infected individuals ex vivo or in naïve single-cell cultures stimulated in vitro. Taking advantage of the co-expression of productive and non-productive Tcra alleles, we demonstrate secondary rearrangement in the TCR $\beta$ locus ex vivo, while the in vitro cultures demonstrated a remarkably high rate of Tcra revision following strong stimulation. Not surprisingly, revision was found to be RAGdependent. Utilizing a model of peripheral deletion of RAG2, we showed that mice deficient in revision showed delayed onset and severity of disease in an experimental autoimmune encephalomyelitis (EAE) model, and also produced a less functional T cell response following challenge with influenza virus. Additionally, in the absence of revision, the dominant $\mathrm{NP}_{366}$ response was compromised in the recall phase while the responses to the minor epitopes were increased. Thus, peripheral TCR revision appears to be a robust process that contributes to optimal $\mathrm{T}$ cell functionality and repertoire diversity during infection but may also contribute to the development of autoimmunity.

\section{2}

Tolerance by deletion and regulatory $T$ cell differentiation arise from different TCR repertoires during two waves of thymic selection

Hu, D.Y. ${ }^{1}$, Singh, M. ${ }^{2}$, Yap, J.Y. ${ }^{1}$, Wirasinha, R.C. ${ }^{3}$, Howard, D. ${ }^{1}$, Archer, S. ${ }^{3}$, Goodnow, C.C. ${ }^{2}$, Daley, S.R. ${ }^{3}$

${ }_{1}^{1}$ The John Curtin School of Medical Research, The Australian National University, Canberra, Australia,

${ }^{2}$ Garvan Institute of Medical Research, Sydney, Australia, ${ }^{3}$ Monash Biomedicine Discovery Institute, Infection and Immunity, Melbourne, Australia

Thymocytes that receive a strong T cell receptor (TCR) signal may be deleted by apoptosis or diverted into the Foxp3+ T-regulatory (Treg) cell lineage. We are investigating whether this divergence is governed by the developmental stage at which thymocytes register a strong TCR signal. Analysis of apoptosis-defective mice suggests that $\sim 50 \%$ of TCR-signalled thymocytes are deleted $<3$ days after the cells proliferate as TCR ${ }^{-10}$ precursors. We characterise these short-lived Helios+ CCR7- PD-1 ${ }^{\text {high }}$ thymocytes as wave 1 of thymic selection. Another subset of Helios+ thymocytes, defined by high CCR7 expression, forms $\sim 5$ days after proliferation and contains cells that upregulate Foxp3 protein 6 - 